Europhysics Letters

PREPRINT

\title{
Frequency-domain study of relaxation in a spin glass model for the structural glass transition
}

\author{
F. RAO ${ }^{1}$, A. Crisanti ${ }^{2}$ And F. Ritort ${ }^{3}$ \\ 1 Department of Biochemistry, University of Zurich, Winterthurerstrasse 190, CH-8057 \\ Zurich, Switzerland. \\ 2 Dipartimento di Fisica, Università di Roma, "La Sapienza" and INFM unità di Roma \\ I and SMC, P. le A. Moro 2, 00186, Rome, Italy. \\ 3 Departament de Física Fonamental, Facultat de Física, Universitat de Barcelona, \\ Diagonal 647, 08028 Barcelona, Spain.
}

PACS. 64.60.-i - General studies of phase transitions.

PACS. 64.70.Pf - Glass transitions.

PACS. 75.10.Nr - Spin-glass and other random models.

\begin{abstract}
We have computed the time-dependent susceptibility for the finite-size mean-field Random Orthogonal model (ROM). We find that for temperatures above the mode-coupling temperature the imaginary part of the susceptibility $\chi^{\prime \prime}(\nu)$ obeys the scaling forms proposed for glass-forming liquids. Furthermore, as the temperature is lowered the peak frequency of $\chi^{\prime \prime}$ decreases following a Vogel-Fulcher law with a critical temperature remarkably close to the known critical temperature $T_{c}$ where the configurational entropy vanishes.
\end{abstract}

A model Hamiltonian or an effective Lagrangian capable of describing relaxation processes in supercooled liquids and structural glasses is difficult to obtain. However, starting with the work of Kirkpatrick, Thirumalai and Wolynes [1,2] in the late 80's, it is now clear that there is a close analogy between some mean-field spin-glass models and structural glasses 3 . The former thus provide a set of microscopical models where glassy dynamics can be studied analytically. The basic simplification occurring in mean-field models is that after averaging over the disorder and making the number of spin very large $(N \rightarrow \infty)$ one is left with a closed set of equations for the two-time correlation and response functions which, above a critical temperature, are basically equivalent to the schematic mode coupling equations introduced by Leutheusser, Götze and others [4] as a model for the ideal glass transition.

In mean-field models the barrier separating different ergodic components diverges in the mean-field limit, hence at the critical temperature $T_{D}$ a real ergodic to non-ergodic transition takes place with diverging relaxation times since barriers cannot be overcome, in agreement with mode-coupling theories (MCT) where $T_{D}$ coincides with the MCT critical temperature $T_{M C T}$. In what follows we will denote $T_{D}$ by $T_{M C T}$. In real systems $T_{M C T}$ still denotes the separation temperature for activated hopping dominated dynamics [5], however, barriers are of finite height and the glass transition appears at $T_{g}<T_{M C T}$ where the typical activation time over barriers is of the same order of the observation time. To go beyond mean-field it is

(c) EDP Sciences 
necessary to include activated processes, a very difficult task since it implies the knowledge of excitations involved in the dynamics.

Recently it has been shown how activated processes in mean-field models could be included just keeping $N$ finite [6], and hence giving support to the scenario of the fragile glass transition developed from spin-glass models. This is not a trivial assumption since it is not a priori clear why excitations in mean-field spin glass models should have similar properties to those of supercooled liquids. This, for example, seems to be the case for the Random Orthogonal model (ROM) [7], but not for the mean-field Potts-Glass model as indicated by recent studies of its finite-size version 8 .

In a series of works the spectral properties of the primary or $\alpha$ relaxation in supercooled liquids has been largely studied by means of dielectric spectroscopy $9,10,11,12,13$. The main conclusion is a there is a three-parameter scaling function that allows to collapse the imaginary part $\epsilon^{\prime \prime}(\nu)$ of the dielectric susceptibility $\epsilon(\nu)$ at different temperatures and for several glass-forming liquids onto a master curve. The master plot is able to reproduce the $\epsilon^{\prime \prime}(\nu)$ data around the relaxation peak $\nu_{p}$ and also at higher frequencies. The bad collapse in the low frequency part has been object of some debates [10,12, however there is no dispute above $\nu_{p}$. The frequency $\nu_{p}$ has a very strong temperature dependence, commonly fitted by a Vogel-Fulcher form $\log _{10}\left(\nu_{p}\right)=\log _{10}\left(\nu_{0}\right)-A /\left(T-T_{0}\right)$, where $T_{0}$ is close to the Kauzmann temperature [14] where the configurational entropy vanishes [15].

In this contribution we compare the frequency-domain analysis of the finite-size ROM with the above scenario. The main motivation for this study was to make a stringent test on the ROM as a possible model for the fragile-glass scenario. The range of temperatures we explore in this paper are all above the mode-coupling transition $T_{M C T}$, therefore we do not expect to find diverging timescales in the large $N$ limit. In the light of the experimental results 9, 10, 11, 12, 13, that also explore the region above $T_{M C T}$, we have decided to focus our attention in this regime in the framework of finite-size mean-field models. Here, we do not address the behavior below $T_{M C T}$ where a $N$ dependence of the frequency response is expected.

The ROM [7] is defined by the Hamiltonian

$$
H=-2 \sum_{i j} J_{i j} \sigma_{i} \sigma_{j}-h \sum_{i} \sigma_{i}
$$

where $\sigma_{i}= \pm 1$ are $N$ Ising spin variables, and $J_{i j}$ is a $N \times N$ random symmetric orthogonal matrix with $J_{i i}=0$. This model was proposed in [7] as a random extension of some nondisordered models describing low autocorrelation binary sequences. Numerical simulations are performed using the Monte Carlo method with the Glauber algorithm for temperatures in the range 0.6 up to 2.0. For $N \rightarrow \infty$ and $h=0$ this model has a dynamical transition at $T_{M C T}=0.536$, and a static transition at $T_{c}=0.256 \ldots$ [7. To study the frequency response we considered a time-dependent field of the form $h(t)=h_{0} \cos (2 \pi \nu t)$, where the time is measured in MC steps and $h_{0}=0.2$ small enough to be within the linear response regime. In our simulations the typical range of $\nu$ was $10^{-6}-10^{-1}$. For each frequency we have measured the complex susceptibility $\chi(\nu)=\chi^{\prime}(\nu)+i \chi^{\prime \prime}(\nu)$ as

$$
\begin{aligned}
& \chi^{\prime}(\nu)=\frac{1}{N M} \sum_{t=1}^{M} \sum_{j=1}^{N} \sigma_{j}(t) \cos (2 \pi \nu t), \\
& \chi^{\prime \prime}(\nu)=\frac{1}{N M} \sum_{t=1}^{M} \sum_{j=1}^{N} \sigma_{j}(t) \sin (2 \pi \nu t) .
\end{aligned}
$$


The number of MC steps $M$ after equilibration was 100 for the largest $\nu$ and up to $10^{7}$ for the shortest $\nu$. We show here results for $N=300$, the finite-size dependence will be discussed below. The choice of $N=300$ is a good compromise between small sample-tosample fluctuations and small barriers height.

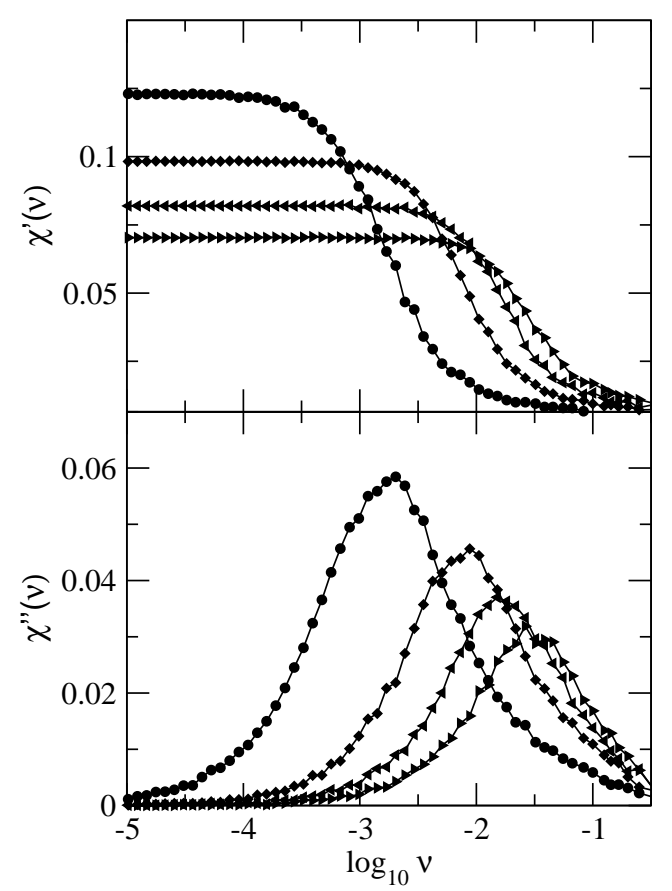

Fig. 1 - The real and imaginary part of the complex susceptibility $\chi^{\prime}$ and $\chi^{\prime \prime}$ as function of $\nu$ for the ROM with $N=300$ at different temperatures. Upper panel top to bottom, lower panel left to right, $T=0.7,0.9,1.1$ and 1.3.

Figure 1 shows the real and imaginary parts of the susceptibility over the available range of frequency. Not all temperatures are reported for a better drawing. The relaxation peak in the imaginary part can be fitted with a log-normal form [16]:

$$
\chi^{\prime \prime}(\nu)=\frac{\Delta \chi}{\sqrt{\pi} \Sigma} \exp \left[-\left(\log _{10} \nu-\log _{10} \nu_{p}\right)^{2} / \Sigma^{2}\right]
$$

where $\nu_{p}$ is the frequency of the peak, $\Sigma$ the width, and $\Delta \chi=\chi_{0}^{\prime}-\chi_{\infty}^{\prime}$, where $\chi_{0}^{\prime}$ and $\chi_{\infty}^{\prime}$ are, respectively, the low and high frequency limit of $\chi^{\prime}(\nu)$.

As the temperature is lowered the peak frequency $\nu_{p}$ decreases, and the width $\Sigma$ broadens. The behavior of $\nu_{p}$ is consistent with the Vogel-Fulcher law $\exp \left[-A /\left(T-T_{0}\right)\right]$ [11. The fit of the frequency peak $\nu_{p}$ for the ROM with the Vogel-Fulcher formula is rather good, see Fig. 2 and gives $A=0.89 \pm 0.06$ and $\ln \nu_{0}=0.64 \pm 0.02 T_{0}=0.28 \pm 0.02$, a value in agreement with the critical value $T_{c}=0.256 \ldots$. We note, however, that data can also be fitted using different expressions such as $\exp \left[-A / T^{2}\right]$ or the Adam-Gibbs formula $\exp \left[-A /\left(T S_{c}(T)\right)\right]$ where $S_{c}$ is the configurational entropy. In particular we note that the data can be also fitted with the formula (CR) $\nu_{p}=\nu_{0} \exp \left[-A \beta_{\mathrm{eff}}(T) / T\right]$ where $\beta_{\mathrm{eff}}(T)=\partial S_{c}\left(e_{\mathrm{is}}\right) /\left.\partial e_{\mathrm{is}}\right|_{e_{\mathrm{is}}=e_{\mathrm{is}}(T)}$ derived from a cooperative scenario of relaxation [17, see Fig. 2] The discrepancy at high temperature is probably due to a poor numerical estimation of the configurational entropy, indeed a similar 
deviation is found using the Adam-Gibbs formula (not reported). We note that the CR formula predicts a crossover form fragile to strong behaviors as the temperature is lowered. However differences among all the above expressions can be appreciated only for very low values of $\nu_{p}$ which are out of our measurements range.

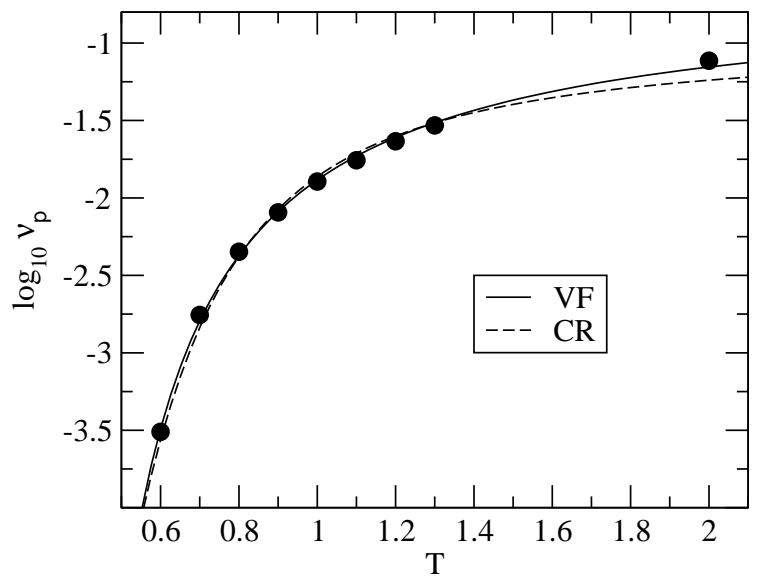

Fig. $2-\log _{10} \nu_{p}$ as function of $T$ for the ROM with $N=300$. The full line is the Vogel-Fulcher law $\nu_{p}=\nu_{0} \exp \left[-A /\left(T-T_{0}\right)\right]$ while the dashed line is the formula $\nu_{p}=\nu_{0} \exp \left[-A \beta_{\text {eff }}(T) / T\right]$ of Ref. [17] with $\beta_{\text {eff }}(T)=\partial S_{c}\left(e_{\text {is }}\right) /\left.\partial e_{\text {is }}\right|_{e_{\text {is }}=e_{\text {is }}(T)}$ evaluated using the results of Refs. [6].

The analysis of the response for glass-former liquids reveals three power laws for $\chi^{\prime \prime}$ [13]: $\chi^{\prime \prime} \sim \nu^{m}$ for $\nu<\nu_{p}, \chi^{\prime \prime} \sim \nu^{-\beta}$ just above $\nu_{p}$ and $\chi^{\prime \prime} \sim \nu^{-\sigma}$ for larger values $\nu \gg \nu_{p}$. The use of Monte Carlo dynamics prevents us from resolving the last behavior due to the discreteness of the time step, nevertheless the first two regimes are clearly seen, as indicated in Figure 3 At higher temperatures $m=\beta=1$ and the relaxation is Debye-like. As the temperature is lowered the value of $\beta$ decreases below 1. It is known that for glass-former liquids $\beta$ and $\sigma$ are related by $(\sigma+1) /(\beta+1)=\gamma$, where $\gamma$ is a constant 13. Furthermore $\sigma$ varies linearly with temperature: $\sigma=B\left(T-T_{\sigma}\right)$ with $T_{\sigma} \simeq T_{0}$ 13, This implies that $\beta=B^{\prime}\left(T-T_{0}\right)+(1-\gamma) / \gamma$. Inserting into this formula the values of $\beta$ obtained for the ROM at various temperatures and the value of $T_{0}$ computed from $\nu_{p}$ we find $\gamma=0.72 \pm 0.02$ the same value found for real liquids 11, 13.

Analyticity of $\chi(\nu)$ and linearity of absorption at asymptotically low frequencies implies that $\chi^{\prime \prime}(\nu) \propto \nu$ for $\nu \ll \nu_{p}[10$. For the ROM with $N=300$ we find $m \simeq 1$ for temperatures down to about 0.8. Below $T=0.8$ significant deviations with $m<1$ are observed. The temperature where $m=1$ breaks down decreases as $N$ increases. Similar deviations have been observed in data from glass-forming liquids and generated some controversy [9, 10, 12 on the reliability of the scaling form proposed by Dixon et al. [9]. Many liquids also posses secondary relaxations which overlap the primary response broadening the peak and leading to deviation from linearity [12. In the case of ROM these secondary relaxations are related to the fact that the barriers separating the low states sampled as the temperature is decreased toward $T_{M C T}$ are not well separated for not too large $N$. Indeed studies of mean-field spinglass models for the structural glass transition shows that in the thermodynamic limit there is no gap between saddles separating local minima with energy above the threshold energy associated with the dynamical transition [18. This is a situation more reminiscent of spinglasses rather than glasses. Indeed both experimental [19] and numerical simulations [19,20] 


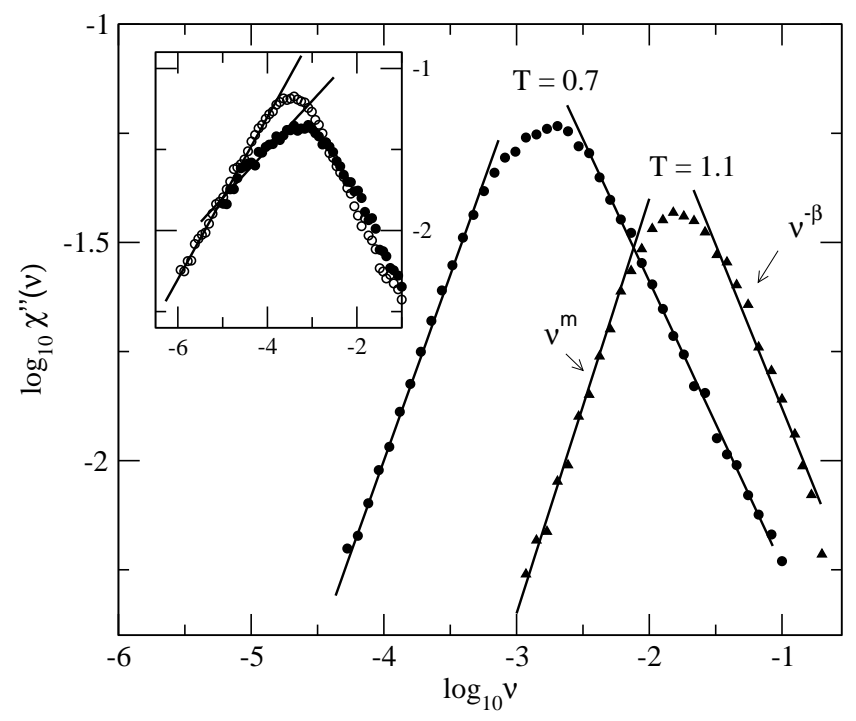

Fig. $3-\chi^{\prime \prime}(\nu)$ for the ROM with $N=300$ for temperatures $T=0.7$ and 1.1. The exponents are $m=1, \beta=1$. for $T=1.1$ and $m=.96, \beta=.71$ for $T=0.7$. Inset: $\chi^{\prime \prime}(\nu)$ at temperature $T=0.6$ for $N=64$ (filled circles) and 300 (empty circles). The lines have slope $m=0.3$ for $N=64$ and $m=0.5$ for $N=300$. The increase of $m$ toward 1 as $N$ grows is clearly seen.

show a broader shape of $\chi^{\prime \prime}(\nu)$ near the peak.

This scenario is supported by a finite-size scaling analysis of the ROM. Indeed we find that for a fixed temperature while $\beta$ is independent on $N$, the value of $m$, when less than 1 , increases toward 1 as $N$ is increased. In the inset of Figure 3 we show $\chi^{\prime \prime}(\nu)$ at $T=0.6$ for systems of size $N=64$ and $N=300$. The increase of $m$ going from 64 to 300 spins is well evident. We now finally address the goodness of the Nagel scaling. Dixon et al 9 ] have shown that all data for the dielectric susceptibility $\epsilon(\nu)$ of different glass-forming liquids and temperatures can be collapsed onto a single master curve by plotting $(1 / w) \log _{10}\left(\epsilon^{\prime \prime}(\nu) \nu_{p} / \nu \Delta \epsilon\right)$ versus $(1 / w)(1 / w+1) \log _{10}\left(\nu / \nu_{p}\right)$, where $\Delta \epsilon=\epsilon^{\prime}(0)-\epsilon^{\prime}(\infty)$ is the relaxation strength measured by the step height of the real part of $\epsilon(\nu), \nu_{p}$ is the peak frequency, and $w$ is the halfmaximum width of the $\epsilon^{\prime \prime}(\nu)$ peak normalized with the corresponding width of the Debye peak: $w_{D} \simeq 1.14$ decades.

Figure 4 shows the Nagel plot for the ROM with $N=300$. The data are from temperatures ranging from 0.6 up to 2.0. For each curve the parameters $\Delta \chi, w=2 \sqrt{2} \Sigma / w_{d}$ and $\nu_{p}$ have been obtained from the log-normal fit of $\chi^{\prime \prime}$ using (4). We see that while the collapse for $\nu>\nu_{p}$ is good for all temperatures, for $\nu<\nu_{p}$ only data with $m=1$ do collapse. As noted in Refs. [12] the optimization of the three parameters through the fitting is essential to have a good collapse of data. For comparison in the inset we report the Nagel plot for the same data done using the parameters read directly from the plot of Figure 1 without any fitting. The improvement through the fitting is evident.

In conclusion, we have shown that the primary relaxation in the finite size mean-field ROM obeys the scaling form typical of glass-forming liquids. Furthermore, the frequency peak of the imaginary part of the complex susceptibility follows the Vogel-Fulcher law with critical temperature $T_{0}=0.28 \pm 0.02$ very close to the critical temperature $T_{c}=0.256 \ldots$, the Kauzmann temperature of the model. All system sizes studied (up to $N=300$ ) lead 


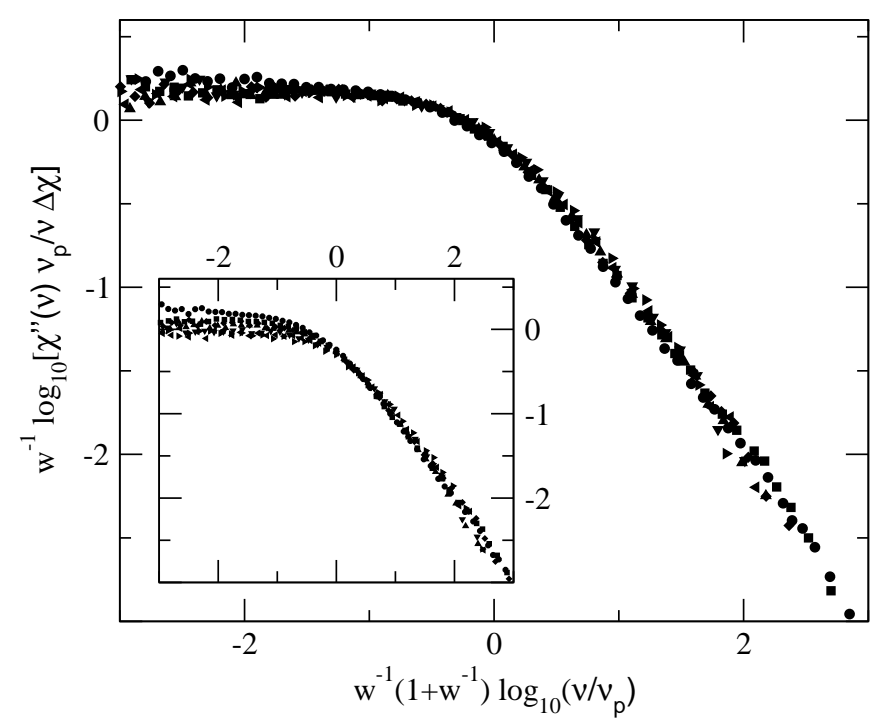

Fig. 4 - The Nagel's scaling for the ROM with $N=300$ and temperatures $T=2.0,1.3,1.2,1.1,1.0$, $0.9,0.8,0.7$ and 0.6. See text for details.

to this value for $T_{0}$. Because we used Monte Carlo dynamics there is a maximum value for the frequency $\sim N$ determined by the discreteness of the elementary time step. As a consequence we are not able to resolve the second scaling behaviors of the imaginary part of the susceptibility $\chi^{\prime \prime}(\nu) \sim \nu^{-\sigma}$ for $\nu \gg \nu_{p}$. Nevertheless by assuming that the exponent $\sigma$ vanishes linearly at $T_{0}$, we obtained for the constant $\gamma$ relating the exponents $\beta$ and $\sigma$ the value $\gamma=0.72 \pm 0.02$, the same found for real glass-forming liquids [1],13. Again this value does not depend on the system size for the sizes we studied. Changing $N$ only modifies the temperature below which secondary relaxations related to barrier crossing breaks the Nagel scaling. Overall, the present results show that finite-size mean-field spin glasses capture the cooperative effects responsible for the relaxational processes observed in glass forming liquids when approaching the mode-coupling temperature. Moreover, to our knowledge, this is the first time that finite-size mean-field spin glasses give a quantitative prediction in agreement with experimental data. The extension of this analysis to the region below $T_{M C T}$ where strong finite $N$ effects are to be observed remains an interesting open problem.

$$
* * *
$$

We acknowledge F. Sciortino and P.Tartaglia for a critical reading of the manuscript. A.C. acknowledges support from the INFM-SMC center. F.R has been supported by project the Spanish Ministerio de Ciencia y Tecnología Grant number BFM2001-3525 and Generalitat de Catalunya. A.C and F.R have also benefited from the Acciones Integradas España-Italia HI2000-0087.

\section{REFERENCES}

[1] T.R. Kirkpatrick and P.G. Wolynes, Phys. Rev. B 36, 8552 (1987). 
[2] T.R. Kirkpatrick and D. Thirumalai D, Phys. Rev. Lett. 58, 2091 (1987); Phys. Rev. B 36, 5388 (1987).

[3] J.P. Bouchaud, L.F. Cugliandolo, J. Kurchan and M. Mezard, in Spin Glasses and Random Fields, A. P. Young ed. (World Scientific, Singapore, 1998), p. 161.

[4] E. Leutheusser, Phys. Rev. A 29, 2765 (1984); U. Bengtzelius, W. Goetze and A. Sjolander, J. Phys. C 17, 5915 (1984); W. Götze in Liquids, Freezing and Glass Transition, J. P. Hansen, D. Levesque and J. Zinn-Justin ed. (North-Holland, 1991), p. 287.

[5] G. Fabricius and D. A. Stariolo, Phys. Rev. E 66, 031501 (2002); B. Doliwa and A. Heuer, Phys. Rev. E 67, 030501(R) (2003); R. A. Denny, D. R. Reichman and J.-P. Bouchaud, Phys. Rev. Lett. 90, 025503 (2003);

[6] A. Crisanti and F. Ritort, Europhys. Lett. 51, 147 (2000); 52, 640 (2000);

[7] E. Marinari, G. Parisi and F. Ritort, J. Phys. A 27, 7647 (1994).

[8] C. Brangian and W. Kob and K. Binder, Europhys. Lett. 53, 756 (2001); J. Phys. A 35, 191 (2002).

[9] P.K. Dixon, L. Wu, S.R. Nagel, B.D. Williams and J.P. Carini, Phys. Rev. Lett. 65, 1108 (1990); Phys. Rev. Lett. 66, 960 (1991); R.V. Chamberlin, Phys. Rev. Lett 66, 959 (1991);

[10] A. Schönhals, F. Kremer and E. Schlosser, Phys. Rev. Lett. 67, 999 (1991); Phys. Rev. Lett. 71, 4096 (1993); N. Menon and S. R. Nagel, Phys. Rev. Lett. 71, 4095 (1993);

[11] N. Menon and S.R. Nagel, Phys. Rev. Lett. 74, 1230 (1995);

[12] A. Kudlik, S. Benkhof, R. Lenk and E. Rössler, Europhys. Lett. 32, 511 (1995); R.L. Leheny, N. Menon and S.R Nagel, Europhys. Lett. 36, 473 (1996);

[13] R.L. Leheny and S.R. Nagel, Europhys. Lett. 39, 447 (1997)

[14] W. Kauzmann, Chem Phys. Rev. 43, 219 (1948)

[15] See, e.g., C. A. Angell, J. Phys. Chem. Solids 49, 863 (1988); Physica D 107, 122 (1997).

[16] L. Wu and S.R. nagel, Phys. Rev. B 46, 11198 (1992).

[17] A. Crisanti and F. Ritort, Philosophical Magazine B 82, 143 (2002).

[18] A. Cavagna, I. Giardina and G. Parisi, J. Phys. A 30, 7021 (1997).

[19] D. Bitko, N. Menon, S.R. Nagel, T.F. Rosenbaum and G. Aeppli, Europhys. Lett 33, 489 (1996).

[20] F. Rao, Master Thesis, University of Rome "La Sapienza", 2001, (unpublished). 